Western University

Scholarship@Western

Electrical and Computer Engineering

Electrical and Computer Engineering

Publications

Department

Summer 8-1-2018

\title{
Grasp and stress analysis of an underactuated finger for proprioceptive tactile sensing
}

Mahyar Abdeetedal

Epson, mahyar.etedal@icloud.com

Mehrdad Kermani Ph.D., P.Eng.

Western University, mkermani@eng.uwo.ca

Follow this and additional works at: https://ir.lib.uwo.ca/electricalpub

Part of the Computer Engineering Commons, and the Electrical and Computer Engineering Commons

Citation of this paper:

M. Abdeetedal and M. R. Kermani, "Grasp and Stress Analysis of an Underactuated Finger for Proprioceptive Tactile Sensing," in IEEE/ASME Transactions on Mechatronics, vol. 23, no. 4, pp. 1619-1629, Aug. 2018, doi: 10.1109/TMECH.2018.2844327 


\title{
Grasp and Stress Analysis of an Underactuated Finger for Proprioceptive Tactile Sensing
}

\author{
Mahyar Abdeetedal, Mehrdad R. Kermani
}

\begin{abstract}
This paper presents the design and evaluation of a new sensorized underactuated self-adaptive finger. The design incorporates a two-degrees-of-freedom (DOF) link-driven underactuated mechanism with an embedded load cell for contact force measurement and a trimmer potentiometer for acquiring joint variables. The utilization of proprioceptive (internal) sensors results in tactile-like sensations in the finger without compromising the size and complexity of the proposed design. To obtain an optimum finger design, the placement of the load cell is analyzed using Finite Element Method (FEM). The design of the finger features a particular rounded shape of the distal phalanx and specific size ratio between the phalanxes to enable both precision and power grasps. A quantitative evaluation of the grasp efficiency by constructing a grasp wrench space is provided. The effectiveness of the proposed design is verified through experimental results that demonstrate the grasp external wrench tolerance, shape adaptability, and tactile capability. All CAD files and ROS package for the proposed underactuated design can be found on https://github.com/mahyaret.
\end{abstract}

Index Terms-Robotic Grasp, Underactuated Mechanism, Mechanism Design.

\section{INTRODUCTION}

A $\mathrm{N}$ industrial gripper is mostly used to manipulate only preplanned objects of similar shape. Small changes in the object shape or weight require the gripper to be modified [1]. There are industrial applications, such as agricultural harvesting in which the target objects, i.e., crops have significant variations in shape and size. In the design of robotic hands, task adaptation capability usually correlates with complex kinematic structures with a high number of degrees of freedom, which may increase the size, control complexity and weight of the device. In addition, in cases where the operation varies from one object to the other, grasp configuration is different for each grasp scenario. Planning new grasp configurations requires contact forces and locations to fulfill the task objectives towards accurate object placement and damage avoidance [2], [3], [4]. Addressing all of these challenges often increases the gripper size and complexity. Tight conditions on space requirements, on the other hand, demand for a compact gripper design. In this paper, an underactuated finger design is proposed which provides tactile-like feedback information without compromising the size and complexity.

A mechanism is needed which can passively adapt to the shape of different objects, without requiring additional actuators and/or sophisticated control strategies. When a robotic mechanism has fewer actuators than the degrees of freedom,

Mahyar Abdeetedal and Mehrdad R. Kermani are with Faculty of Electrical and Computer Engineering, Western University, London, Ontario, Canada mabdeete@uwo.ca, mkerman2@uwo.ca

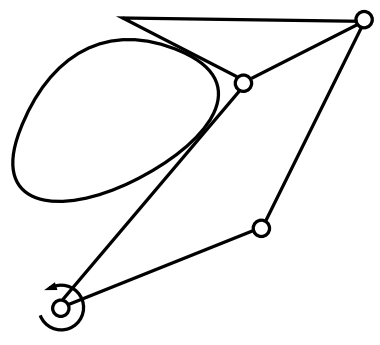

(a)

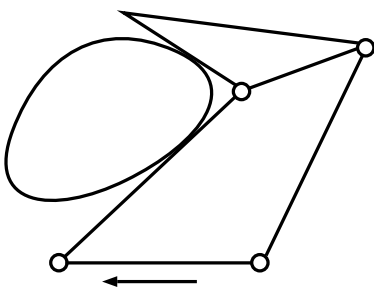

(b)
Fig. 1. Two different actuation approaches of a 2-DOF underactuated finger. (a) actuation using a revolute joint, and (b) actuation using prismatic joint.

it is known as an underactuated mechanism. An underactuated robotic hand provides passive motions imposed by the object geometry, the transmission mechanism and passive elements such as springs or compliant joints. Soft Gripper is the first widely known underactuated gripper prototype [5]. This gripper consists of multi-links and a series of pulleys that are actuated by a pair of wires with neither control nor feedback sensors. There are other underactuated fingers which are based on tendon-actuated mechanism [6], [7], [8]. Most tendonactuated mechanisms are limited to small grasping forces that are deteriorated by friction and elasticity [9]. There are a number of other important underactuation approaches for robotic hands, e.g., eigen-grasps [10], parallel structure based [9] and adaptive synergies [11], [12]. Another transmission approach in underactuated fingers is based on linkage-actuated mechanism which is known to have structural robustness and high force insertion capability [13], [14]. Fig. 1] shows two implementations of a single actuation using revolute and prismatic joints with generalized torque distribution among two phalanges. In this paper, the design, development, and evaluation of a sensorized finger based on link-driven actuation mechanism are presented.

The intrinsic ability of link-driven underactuated fingers to adapt to an object shape makes grasping of unknown geometries possible. In an underactuated power grasp, the robotic hand wraps around the object and provides a robust grasp. An alternative approach in the design of underactuated hand is to enable precision grasp. In this approach, the fingers are designed such that the fingertips are mostly in contact with the object. In both cases, the form adaptability of linkdriven underactuated fingers is dictated by the shape of the object, not by the motion of the actuator. In other words, since fingers have one actuator but several contact forces, the contact 
forces can be uncontrollable and dependent. Therefore, the knowledge of contact forces becomes exceedingly important for evaluating a grasp for a particular task. One such evaluation is to characterize a grasp based on the set of external wrenches that the grasp can withstand during object manipulation. This is known as Grasp Wrench Space (GWS) [15]. Having additional sensors for obtaining contact positions and forces becomes an integral part of grasp synthesis. The ability to extract position and force information of the fingers is one of the main advantages of the proposed design in this paper.

Tactile sensors can be used to acquire contact forces and positions [9], [16]. Tactile feedback has a wide range of applications from robotic hand to teleoperated devices [17]. The data from tactile sensors can serve in assessing grasp stability, performing object recognition, detecting slippage, and detecting collisions [18], [19], [20]. Typically, tactile sensors, also known as robot skins consist of an array of sensors that cover an area of a finger or hand to provide contact positions and forces [21]. While robot skins provide an operative means of measuring forces and positions, their construction is often sophisticated and prohibitively costly [22]. For these reasons, the application of robot skins in practice has been somewhat limited.

Another approach for obtaining contact information is using proprioceptive (internal) sensors instead of exteroceptive sensors. Proprioceptive sensors are used to determine the location of contact points [23], [24], [25]. For instance, negative torque compensation at the inter-phalanx joints of the finger can provide a rough estimation of the contact positions with no information about forces [26], [27]. In this paper, a new design that provides both contact positions and forces is proposed. Such design addresses the challenges of using tactile sensors and the shortcomings of inaccurate data estimation by incorporating a load cell and a trimmer potentiometer. Our design allows for a low cost, yet reasonably accurate force and position measurements. The main contributions of this paper are as follows

- A new approach is introduced for obtaining tactile information. Our approach is based on combining the data obtained from a potentiometer and a load cell. Both experimentally and theoretically, it is shown that the suggested approach is capable of contact position estimation.

- A new modification for the formulation of the transmission matrix for an underactuation mechanism is provided. The new transmission matrix is used to derive the Jacobian matrix which is needed for our contact estimation method. Unprecedentedly in the underactuation literature, the provided Jacobian matrix considers contact model, and it is also applicable to both link-driven prismatic and revolute underactuated mechanisms.

- A new sensorized underactuated finger is designed, and 3D printed. The design approach for the load cell placement is examined by stress analysis of the finger using FEM. The embedded load cell not only enables the contact point estimation but also facilitates the grasp of fragile objects such as egg. The grip robustness is visualized and evaluated by forming the Grasp wrench space of the prototyped two-finger gripper. Furthermore, the estimation of unknown object centroid is implemented via contact estimation, joint variable measurement, and self-adaptation of the finger.

The structure of this paper is as follows: Section II presents the kinetostatic analysis of underactuated mechanism. Section III provides the proposed contact estimation method. Section IV provides the underactuated finger design. Section V studies the validity of the presented approach experimentally using prototyped underactuated fingers. Section VI discusses possible limitations of the proposed method. Section VII concludes the paper and provides future research directions.

\section{Kinetostatic Analysis}

To define the relevant velocity kinematics and force transmission properties of a robotic hand, the finger Jacobian, $J$, needs to be defined. The transpose of finger Jacobian matrix is defined as a mapping between the load of the finger joints and the forces and moments at contact points. Kinematically, the finger Jacobian matrix can be expressed as a mapping between the finger joint velocities to the twists of the hand at contact frames. Usually, in the underactuation literature, the finger characterization is done independently from the grasped object; and the underactuated mechanism is considered to be actuated by a revolute joint [9].

In this section, two important concepts to define the finger Jacobian matrix are integrated. First, both cases of revolute and prismatic joints in underactuated fingers are considered in the definition of the Jacobian matrix. Fig. 1 shows two different approaches of actuating a two-DOF underactuated finger using a revolute joint or a prismatic joint. In both cases, the geometry of the object governs the closure of the fingers where each phalanx activates its adjacent phalanx until full finger closure is formed around the object. The second concept integrated in the definition of the Jacobian matrix is the contact model. The contact model is important for determining the grasp capabilities. Three common contact models are considered in this paper.

The Jacobian matrix can be constructed using three important matrices for grasp characterization. The first matrix is $\tilde{J}$ which relates the velocity of all contact points to joint velocities, i.e., $v_{c, f i n}=\tilde{J} \dot{q}$ where $v_{c, \text { fin }}$ is the contact twist on the finger, and $q$ is the phalanx joint coordinates. Defining $\theta_{i}$ as the $i^{\text {th }}$ joint angle, $l_{i}$ as the lenght of the $i^{\text {th }}$ link, and $c_{i}$ as the position of the contact point $i$, one can derive $\tilde{J}$ using Plücker coordinates of the axes of the joints [28] for the linkage-based underactuated manipulator shown in Fig. 2 as follows,

$$
\tilde{J}=\left[\begin{array}{cccc}
c_{1} & 0 & \ldots & 0 \\
0 & 0 & \ldots & 0 \\
1 & 0 & \ldots & 0 \\
\hline n_{21} & c_{2} & \ldots & 0 \\
t_{21} & 0 & \ldots & 0 \\
1 & 1 & \ldots & 0 \\
\hline \ldots & \ldots & \ldots & \ldots \\
\ldots & \ldots & \ldots & \ldots \\
\ldots & \ldots & \ldots & \ldots \\
\hline n_{n 1} & n_{n 2} & \ldots & c_{n} \\
t_{n 1} & t_{n 2} & \ldots & 0 \\
1 & 1 & \ldots & 1
\end{array}\right]
$$




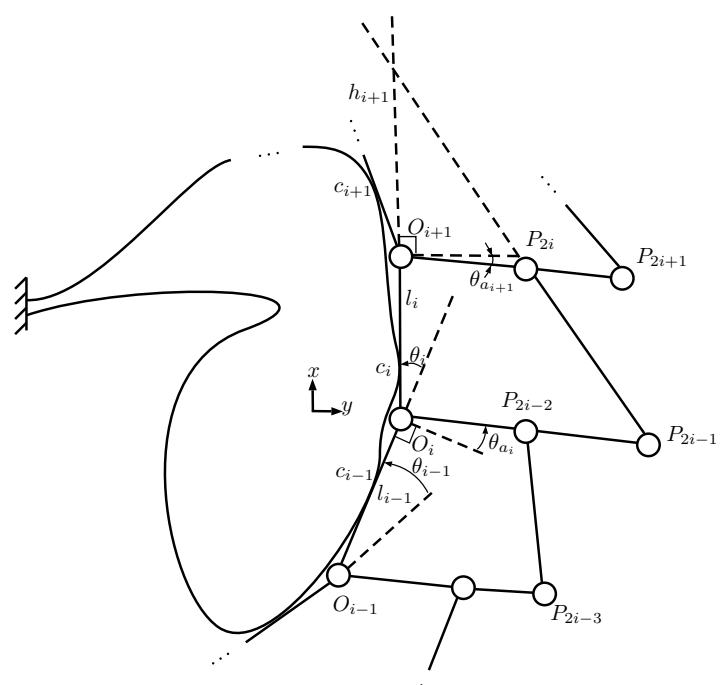

Fig. 2. Detailed modeling of a link-driven finger in contact with a general object with unknown geometry.

where $n_{i j}=c_{i}+\sum_{k=j}^{i-1} l_{k} \cos \left(\sum_{m=k+1}^{i} \theta_{m}\right), j<i$ and $t_{i j}=$ $\sum_{k=j}^{i-1} l_{k} \sin \left(\sum_{m=k+1}^{i} \theta_{m}\right), j<i$. One should note that a rotation in the first joint, i.e., $q_{1}$, does not affect the kinematic configuration of the linkage-based underactuated system.

The second matrix is the transmission matrix, $T$ which relates the input velocity vector, $\dot{\theta}$, to joint velocity vector whose elements are the derivatives of the phalanx joint coordinates, $\dot{q}$, i.e.,

$$
\dot{q}=T \dot{\theta}
$$

The details of the development of matrix $T$ is discussed in [9]. In this paper, this matrix is modified to include the actuation type as follows,

$$
T^{T}=\left[\begin{array}{cc}
\rho & 0_{n-1}^{T} \\
-\frac{h_{2}}{h_{2}+l_{1}} & \\
\cdots & I_{n-1} \\
-\prod_{i=2}^{n} \frac{h_{i}}{h_{i}+l_{i-1}} &
\end{array}\right]
$$

where $h_{i}$ is the signed distance from $O_{i}$ to the intersection point of two lines, $\left(O_{i-1} O_{i}\right)$ and $\left(P_{2 i-2} P_{2 i-3}\right)$ (see Fig. 2). In this matrix, $\rho$ specifies the actuation type of the underactuated mechanism. An underactuated finger with a revolute joint is represented with $\rho=1$, and a prismatic actuation is represented with $\rho \simeq c_{1}$. The arc result from the first joint variable is expressed as $\rho \dot{\theta}_{1}$. The approximation used for prismatic actuation is based on the fact that a curve in a plane can be in general represented with piecewise linear approximation. The length of each linear segment can be calculated using the Pythagorean theorem in Euclidean space. Here, the arc that is shaped by each phalanx rotation is approximated with a line equivalent to the prismatic joint variable change. Note that the virtual work principle can be also used for considering prismatic actuation in an underactuated mechanism [29].

The third matrix is the contact model matrix which selects a number of components of the relative contact twist and sets them to zero. Three different contact models are commonly used in grasp modeling, namely, point contact
TABLE I

SELECTION MATRIX FOR PLANAR CONTACT $i$

\begin{tabular}{ll}
\hline \hline Model & $H_{i}$ \\
\hline \hline Point contact without friction & {$\left[\begin{array}{lll}1 & 0 & 0\end{array}\right]$} \\
Hard and soft finger & {$\left[\begin{array}{lll}I_{2 \times 2} & \mathbf{0}\end{array}\right]$} \\
\hline \hline
\end{tabular}

without friction, hard finger, and soft finger. To obtain a complete Jacobian matrix, each particular contact model with suitable components of the contact twists between the fingers $\left(v_{i, f i n}\right)$ and the object $\left(v_{i, o b j}\right)$ must be considered. The contact model can be expressed as, $H\left(v_{c, f i n}-v_{c, o b j}\right)=0$ where $H=\operatorname{BlockDiag}\left(H_{1}, \ldots, H_{n_{c}}\right)$, in that $H_{i}$ is defined for the $i^{\text {th }}$ contact model as in Table [I The contact model matrix $H$ selects suitable components of the contact twist and sets them to zero. Having determined a contact model, the complete Jacobian matrix is given by,

$$
J=H \tilde{J} T
$$

\section{Contact Point Estimation}

In this paper, a method to estimate the location of the contact points using the definition of the Jacobian matrix is provided. Considering the dual view of the Jacobian matrix, i.e., $\tau=J^{T} F$ where $\tau$ is the actuation vector and $F$ is the contact force vector, the joint torque of the first finger can be represented as follows,

$$
\tau_{a}=\rho c_{1} f_{c_{1}}
$$

where $\tau_{a}$ is the generalized actuation torque of the first (actuated) joint, and $f_{c_{1}}$ is the first contact force. Other joints are represented as,

$$
\tau_{k}=\left(-\prod_{i=2}^{k} \frac{h_{i}}{h_{i}+l_{i-1}} n_{k 1}+c_{k}\right) f_{c_{k}}
$$

where $\tau_{k}$ is the actuation torque of the $k^{\text {th }}$ joint, and $f_{c_{k}}$ is the normal contact force on the $k^{\text {th }}$ phalanx. In quasistatic manipulation, the acceleration of the mechanism is negligible. Therefore, at each state of grasping from the moment that the proximal phalanx makes a contact with the object to the complete closure of the finger at the distal phalanx contact, all forces and torques are assumed to be in balance. Additionally, in a link-driven underactuated robot in which low stiffness springs are used to hold the structure, the input torque vector exerted by the actuator and springs must meet the following conditions,

$$
\tau=\left[\begin{array}{c}
\tau_{a} \\
\tau_{2} \\
\cdots \\
\tau_{n}
\end{array}\right]=\left[\begin{array}{c}
\tau_{a} \\
-K_{2} \Delta \theta_{2} \\
\ldots \\
-K_{n} \Delta \theta_{n}
\end{array}\right]
$$

where $\tau_{a}$ is the generalized input actuation torque, $\tau_{i}$ is the $i^{\text {in }}$ joint torque, and $K_{i}$ is the $i^{\text {th }}$ spring constant. In this paper it is assumed that each finger makes contacts with the object with every phalanxes which is the case for most power grasps. It is also assumed that the proximal phalanx makes the first contact 
with the object at the moment of gripper closure. This is a valid assumption since in a link-driven underactuated finger each phalanx is only activated after its preceding phalanx has made a contact. The second assumption limits the proposed method to contact estimation for power grasps. Under these assumptions, one can obtain contact position estimation by combining (5), (6), and (7). In our proposed design, the trimmer potentiometer provides $\Delta \theta_{i}$ for the $i^{\text {th }}$ joint and the $i^{\text {th }}$ embedded load cell provides the contact force on the $i^{\text {th }}$ phalanx. By equating (5) and (7) at the moment of first contact and repeating the procedure for other phalanxes using (6), all contact locations can be obtained.

\section{UndERACTUATED FINGER DESIGN}

\section{A. Design Properties}

To validate our proposed solution for contact location estimation, a link-driven underactuated finger was designed and built as a testbed (The CAD files for the design can be found on https://github.com/mahyaret/SUF). The design was kept small and straightforward to facilitate manufacturing with rapid prototyping technology and fewer parts to assemble. The finger was equipped with position and force sensors while keeping the design compact. A 3D model of the finger is shown in Fig. (3). The total length of the finger is $8 \mathrm{~cm}$, with a maximum width of $3 \mathrm{~cm}$ except at the tip that is reduced to $2 \mathrm{~cm}$. In this design, $l_{1}=5.5 \mathrm{~cm}$, and $h \simeq 3.4 \mathrm{~cm}$ (at $\theta_{3}=0$ ). The positive distance $h$ at rest position is considered to be equal to the length of the distal phalanx. Hence, any contact point on the distal phalanx falls below the equilibrium point which results in finger closure. The first joint can rotate $60^{\circ}$, and the second joint can rotate $80^{\circ}$.

In the design of the finger, mechanical properties of the material were considered to calculate a suitable preloading condition of the spring that would prevent any undesirable motion of the second phalanx due to weight and/or inertial effects, and also would avoid hyperflexion of the finger. The preloaded springs keep the fingers from unintended motion until the grasp sequence is completed. Since these springs oppose the actuator force, the smallest possible stiffness sufficient to keep the finger from collapsing were selected. Moreover, the tip of the finger is designed to be rounded which can roll on the object. Rolling on the object prevents the distal phalanx to guide the object inward while activating the other joint. As a result, bending of the finger happens in precision grasping as well as power grasping.

\section{B. Sensors Placement}

In a link-driven underactuated finger, one joint variable can be used to obtain the values of other joint variables due to their kinematic dependencies. In the proposed design, a trimmer potentiometer is used to measure joint angles. The two ends of the potentiometer were fixed to the first and second phalanxes, respectively. By reading the second joint angle and kinemtatic relations of the two joints, the first joint angle can be obtained.

While measuring joint angles is a simple task, integrating a load cell in the finger design for measuring contact forces is quite challenging. First, there is minimal space available

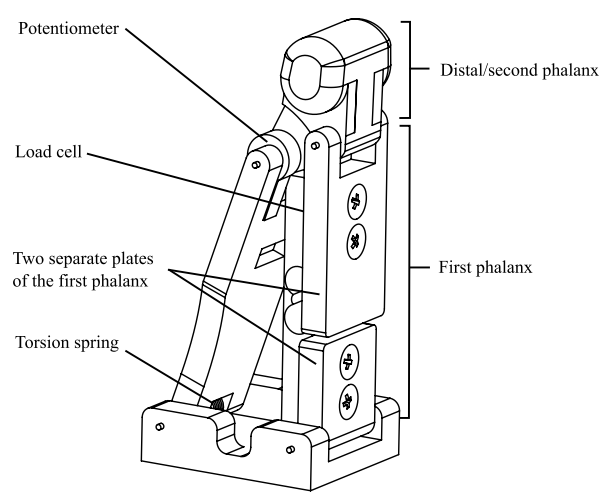

Fig. 3. Underactuated finger packed with a load cell and a potentiometer. Two separate plates attached to the load cell form the proximal phalanx. The rounded fingertip allows finger to be bent in precision grasping. All CAD files for the proposed underactuated design can be found on https://github. com/mahyaret/SUF

in the finger that can be used for the load cell placement. Second, the load cell has to be placed in a position that can read meaningful force data. In this paper, these challenges are addressed using an integrated design approach in which the load cell is used as a part of the proximal phalanx (see Fig. 4). The proximal phalanx includes two separate pieces that are joined together using the load cell. This configuration allows the load cell experience maximum force-induced strains freely while providing structural support for the finger. The gray area in Fig. 4 shows the effective length of the proximal phalanx that measures contact forces. As seen, about $60 \%$ of the proximal phalanx length can be used for force measurement. This measurement length has been achieved by designing the lower part of the proximal phalanx with the smallest size possible.

The proposed design of the finger also allows for measuring both dynamic (during the grasp) and quasi-static contact forces. As the finger starts interacting with an object and bends towards grasping (wrapping around) the object, the load cell starts reading the contact force. The measured contact force is directly related to the stiffness of the spring and the induced strain due to contact. At the same time, when the finger is fully bent, the load cell continues to measure the contact force since one end of the load cell is fixed with respect to the based of the finger via the lower part of the proximal phalanx.

\section{Stress Analysis}

The proposed design of the finger allows for the load cell to experience maximum contact force. This requirement is achieved by using two separate parts (attached with the load cell) in the proximal phalanx. Selecting an optimum length of each part ensures high-stress exertion on the load cell.

To demonstrate the benefit of the proposed design, Fig. 5 compares two cases where a $1 \mathrm{~N}$ contact force is applied at the same location on the proximal phalanx in two different structures. Fig. 5(a) depicts our proposed design with two separate plates joined via the load cell sensor. On the other hand, in Fig. 5.b) the proximal phalanx of the finger consist 


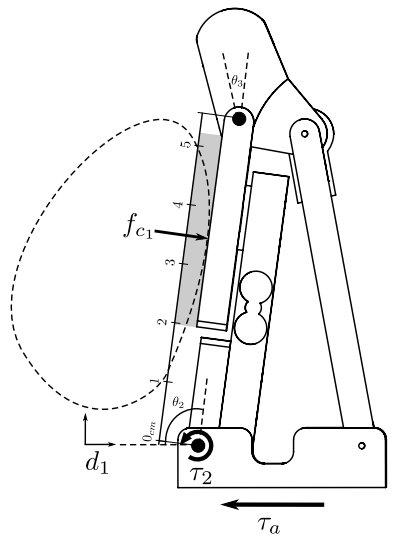

Fig. 4. Generalized actuation, $\tau_{a}$, moves the finger toward the object. Contact force $f_{c_{1}}$ results in finger closure which is opposed by the spring and results in $\tau_{2} . d_{1}$ is the first joint variable, $\theta_{2}$ is the first unactuated joint variable, and $\theta_{3}$ is the second unactuated joint variable. The proximal phalanx is graduated and the gray area (on the second part of the proximal phalanx) is the effective measuring length (roughly $60 \%$ of the proximal phalanx length).

of one piece attached to the load cell. Looking at the stresses experienced in each design, it is obvious that the proposed design provides much higher reading (about 2.5 times higher). The maximum stress $\left(2.29 \times 10^{6} \frac{\mathrm{N}}{\mathrm{m}^{2}}\right)$ will be seen at the location of the strain gauges inside the load cell. In contrast, in the other structure with a single plate screwed to the load cell, much less stress is experienced by the load cell for the same amount of contact force. This analysis demonstrates the rationale behind using two separate plates for holding the load cell.

Another feature of the design is to enable precision grasping while reading contact forces without using tactile sensors. As pointed out previously, an underactuated finger can be designed to be capable of applying precision grasp with the fingertip in contact with the object. However, to measure contact forces, all previous designs in the literature are based on tactile sensors. In the proposed design, a different approach is taken. The rounded tip of the finger is exploited to enable transferring all contact forces to the second joint. This interaction between the first and second join not only enables precision grasping possible but also provide contact forces in precision grasping. Fig. 5. (c) demonstrates this concept in which a contact force at the fingertip is observable through the load cell measurements.

\section{RESUlTs}

In this section, experimental results are provided that validate the capability of the fingers in conducting power grasping as well as precision grasping. In addition, the ability of the proposed finger design for regulating contact forces in handling fragile objects is demonstrated. The contact forces allow visualizing GWS for demonstrating the strength of a sample grasp. Additionally, the capability of the designed fingers in measuring contact locations has been experimentally validated. Combining force and position information allows for obtaining object centroid and providing an estimation of the object shape.

\section{A. Experimental Setup}

Kuka Light-Weight Robot (LWR) IV and CRS Robotics underactuated gripper were used for evaluating the performance of the designed underactuated fingers. Fig. 6 shows this configuration. To exploit the capabilities of Kuka LWR controller along with peripheral tools and sensors, an opensource KUKA UI is developed (https://github.com/mahyaret/ KUKA-UI). This is a comprehensive computer interface that provide seamless integration and synchronous control of National Instruments Data Acquisition board USB-6229 (gripper controller) and PhidgetBridge 4-Input (sensor driver) with Kuka Controller. PhidgetBridge 4-Input was used for acquiring load cell signals which then were filtered by a Kalman filter (integrated in the program) and fed back to the gripper controller. The program was developed based on Kuka Fast Research Interface (FRI) to enable real-time control of the robot. Type II Reflexxes Motion Library was used to generate an online trajectory for Kuka LWR in different control modes.

Shear load cells manufactured by Phidgets were used. The load cells nominal weight capacity were 780 g with 390 $\mathrm{mg}$ maximum repeatability error, $390 \mathrm{mg}$ non-linearity, and $390 \mathrm{mg}$ hysteresis. Load cells were calibrated for measuring contact points using an ATI 6-axis force/torque sensor. The calibration was done for different contact points including precision and power grasping. The data from potentiometer was acquired using the same driver used for the load cells. This driver was interfaced to our developed software.

The matrices $H, \tilde{J}$, and $T$ for the two fingers actuated by CRS Robotics gripper were developed as described in Section II (see 1, 3), and Table I), i.e.,

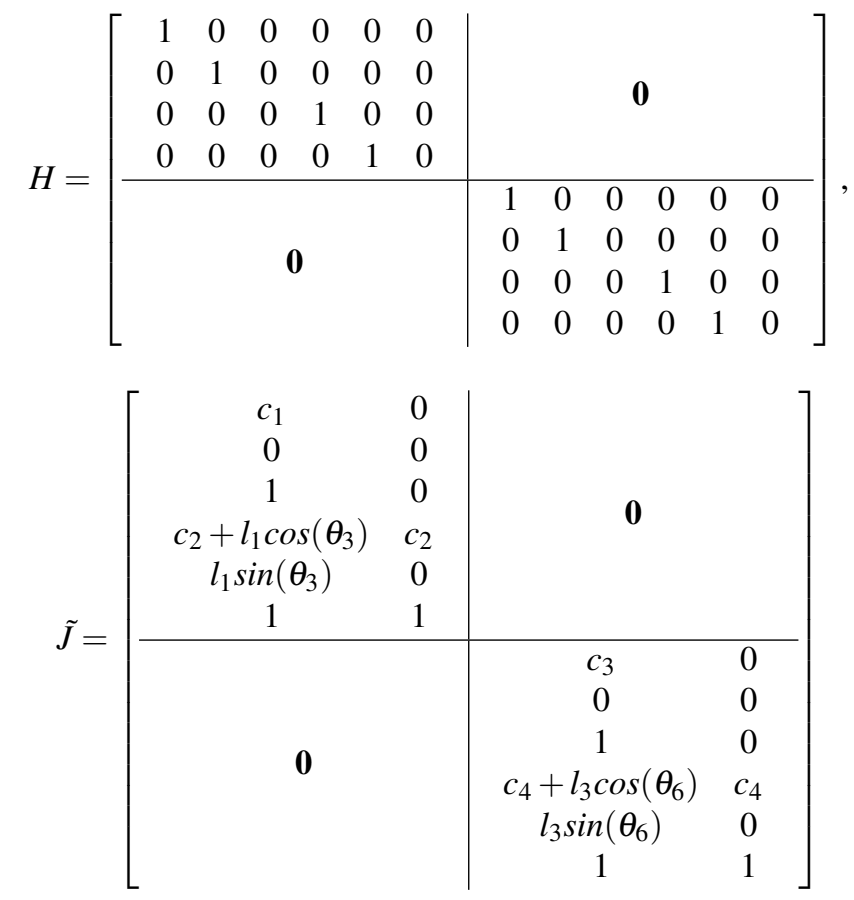

where $c_{1}$ and $c_{2}$ are the first and second contact points on one finger, $c_{3}$ and $c_{4}$ are third and forth contact points on the other finger, $\theta_{3}$ is the second joint variable on one finger, $\theta_{6}$ is the second joint variable on the other finger, and $l_{1}=l_{3}=5.5 \mathrm{~cm}$ in each finger, respectively. The relation between the input 


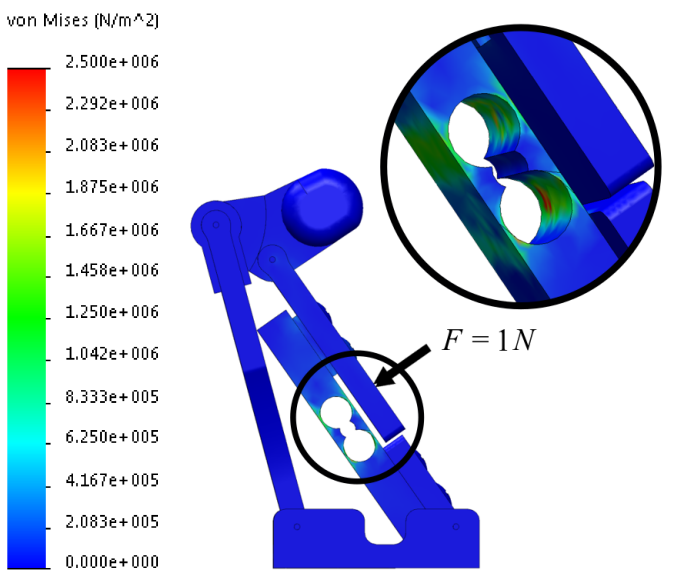

(a)

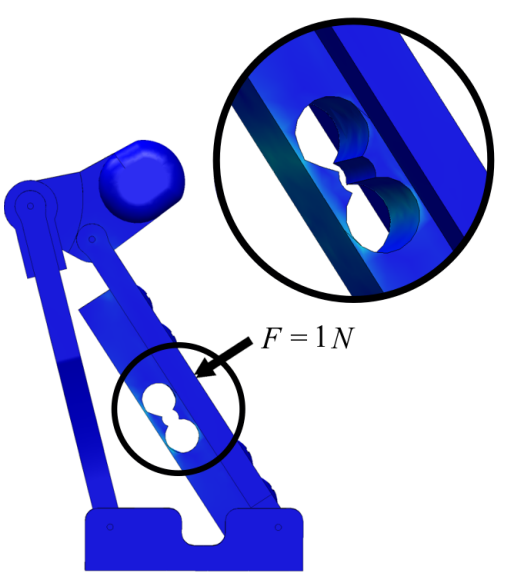

(b)

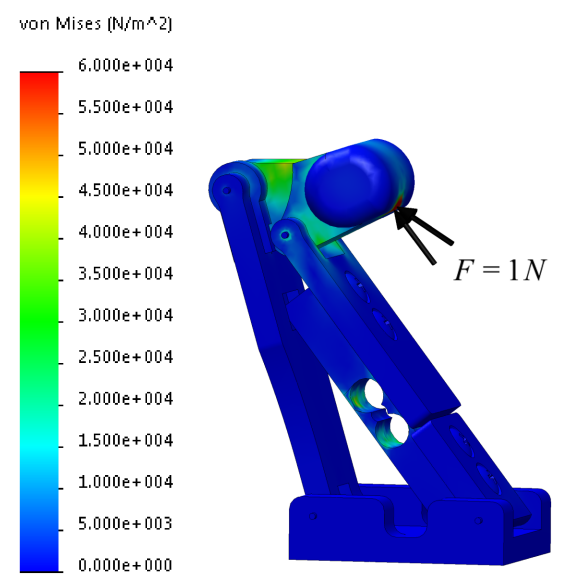

(c)

Fig. 5. Von Mises stress analysis for $1 \mathrm{~N}$ force. The stress analysis is done for a 3D printed finger with acrylonitrile butadiene styrene (ABS) plastic material (\%100 density), and Aluminium load cell made of alloy 1060. Fine meshing was done automatically using Solidworks ${ }^{\mathrm{TM}}$, (a) two separate plates are screwed to the load cell, such structure transfers high stress $\left(2.29 \times 10^{6} \frac{\mathrm{N}}{\mathrm{m}^{2}}\right)$ to the strain gauges on the load cell, (b) a single plate screwed to the load cell, such structure damps the stress experienced by the load cell $\left(6.25 \times 10^{5} \frac{\mathrm{N}}{\mathrm{m}^{2}}\right)$ for the same amount of contact force, (c) $1 \mathrm{~N}$ force is inserted on the fingertip evenly distributed on a $1 \mathrm{~mm}$ square, the fingertip contact force can be measured by load cell.

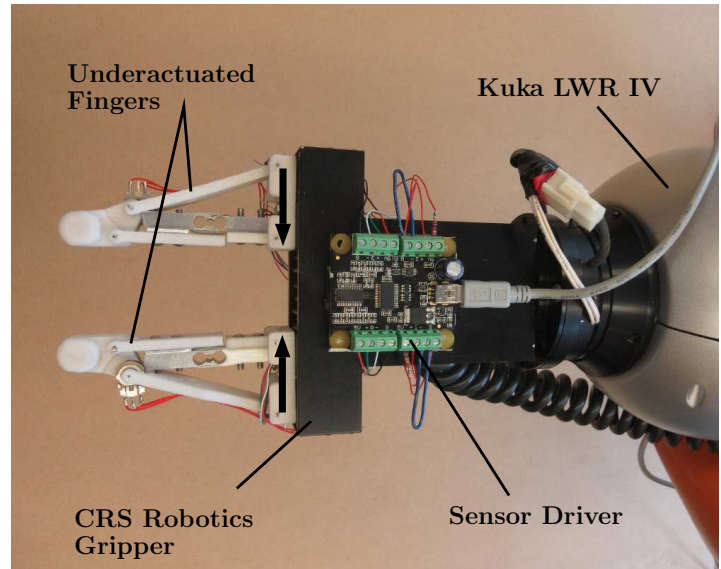

Fig. 6. Experimental hardware setup. A single driver is used for collecting data from two load cells and two potentiometers.

velocity vector to the derivatives of the joint variables, i.e., the transmission matrix in (3), is as follows,

$$
\left[\begin{array}{c}
\dot{\theta}_{2} \\
\dot{\theta}_{3} \\
\dot{\theta}_{5} \\
\dot{\theta}_{6}
\end{array}\right]=\left[\begin{array}{cc|cc}
c_{1} & -\frac{h_{1}}{h_{1}+l_{1}} & & \mathbf{0} \\
0 & 1 & & \\
\hline & \mathbf{0} & c_{3} & -\frac{h_{2}}{h_{2}+l_{3}} \\
& & 0 & 1
\end{array}\right]\left[\begin{array}{c}
\dot{d}_{1} \\
\dot{\theta}_{3} \\
\dot{d}_{4} \\
\dot{\theta}_{6}
\end{array}\right]
$$

where $h_{1}=h_{2} \simeq 3.4 \mathrm{~cm}$ for first and second fingers at rest position (when $\theta_{3}=\theta_{6}=0$ ), and $d_{1}$ and $d_{4}$ are the prismatic joint variables of the fingers.

\section{B. Shape Adaptability}

To test the adaptability of the gripper, a broad range of objects were grasped. In general, the underactuated fingers performed well in grasping different object categories. Examples of grasps are shown in Fig. 77 The rounded design of the second phalanx resulted in bending of the finger even

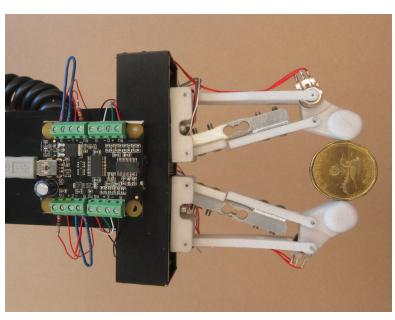

(a)

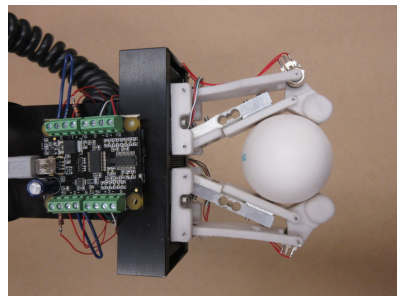

(c)

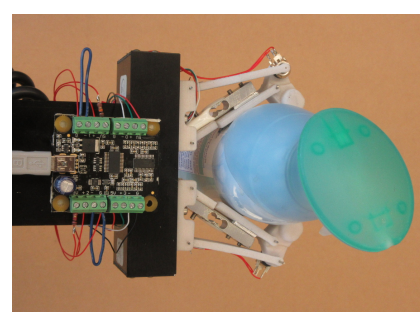

(b)

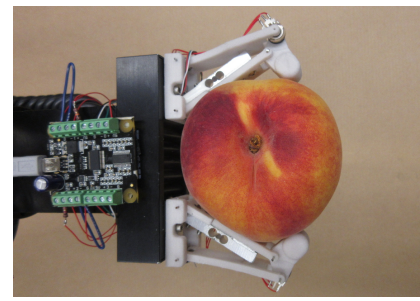

(d)
Fig. 7. Adaptability of the designed finger. (a) Precision grasp of a coin. (b) Power grasp of a spray bottle. (c) Power grasp of an egg. (d) Power grasp of a peach.

in precision grasping. Figure $7 \mathrm{~h}$ shows a precision grasp, in which the second phalanx was bent and contact forces were measured. The experiments carried out with the use of load cell sensors showed that these sensors were capable of achieving fragile objects grasping. The hand was able to grasp very fragile objects, such as eggs (see Fig. 7k) while regulating its contact force. The small width of the fingers and the size of the hand facilitate manipulation of objects in constrained environment such as harvesting fruit and vegetable.

\section{Force Control}

A CRS Robotics gripper was used in our experiments to provide prismatic actuation to the fingers and to achieve force 

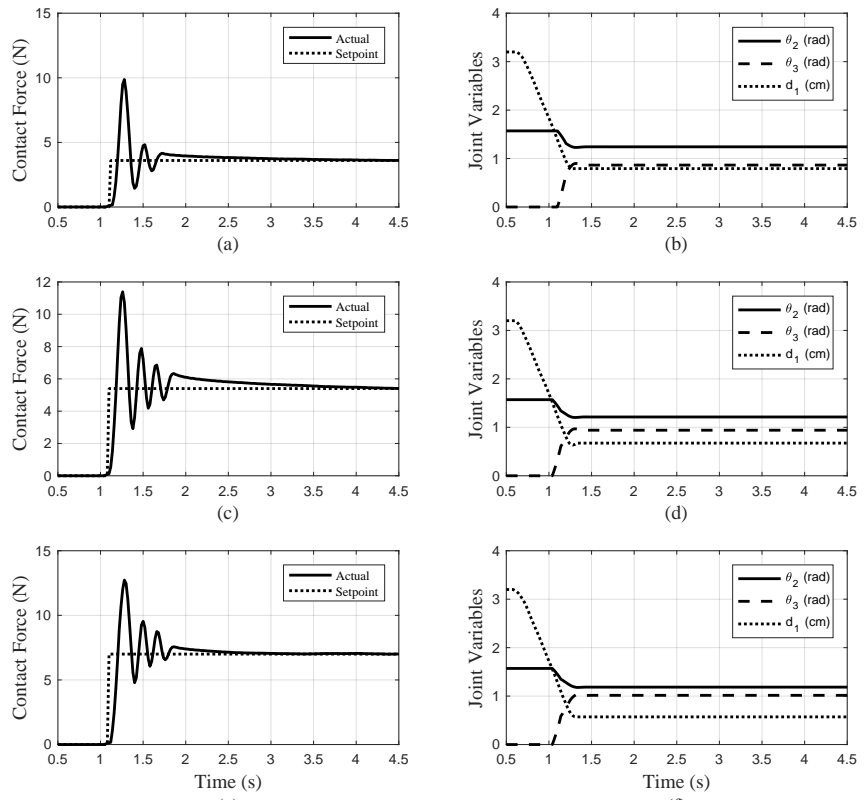

(e)

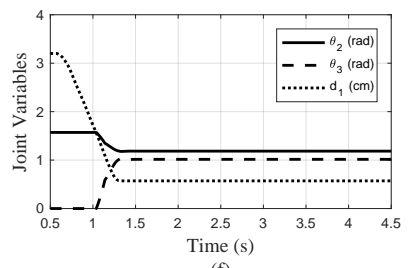

(f)

Fig. 8. Contact forces and joint variables where $d_{1}$ is the first joint variable $\theta_{1}$ is the first unactuated joint variable, and $\theta_{2}$ is the second unactuated joint variable. (a), (c) and (e) are force regulation for $3.5 \mathrm{~N}, 5.5 \mathrm{~N}$, and $7.0 \mathrm{~N}$, and (b),(d), and (f) are the second joint angles.

control using load cells that provide force feedback. The strain gauge based nature of the force sensor makes noise unavoidable. Hence, a PID controller enhanced with Kalman filter was used to regulate contact forces. Samples of the force the regulations are shown in Fig. 8 a for for $f_{c_{1}}=3.5 \mathrm{~N}, 5.5 \mathrm{~N}$, and $7 \mathrm{~N}$, respectively. Respective joint variables for these force values are also illustrated in Fig. 8p. As observed, the velocity prior to the impact translates to high force overshoots. The force overshoots can also be attributed to static friction of the joints and the stress of the torsion springs. The fusion of the position data and force readings is expected to better deal with the force overshoots and contact detection. This conjecture, however, requires further investigation.

\section{External Wrench Tolerance}

In grasp analysis, the knowledge about disturbance tolerance is always a notion of interest. The Grasp Wrench Space (GWS) is defined as the space of wrenches that the grasp can tolerate. This space is equal to the convex hull of the Grasp matrix that is computed using the Quickhull algorithm [30]. Fig. 9 shows the grasp configuration for a four-contact grasp example performed using two of the designed fingers. The GWS for the given configuration is shown in Fig. 10 a,b in the presence of friction with 0.2 and 0.5 friction coefficients, respectively. It is clear from Fig. 10a,b that GWS is highly dependent on the friction coefficient.

The ability of wrench exertion is highly dependant on friction. Assuming that the value of the wrench that needs to be tolerated for the object is known, one must express this wrench in terms of the friction forces and associated normal contact forces. To this end, the nonlinear Coulomb friction cone is often approximated with an $n_{s}$-sided pyramid. A given contact force is then decomposed as a positive linear combination of the force vectors spanning the $n_{s}$-sided friction pyramid, i.e.,

$$
f_{c_{i}}=\sum_{i=1}^{n_{s}} \alpha_{i} f_{i}, \quad \text { for } \quad \alpha_{i} \geq 0
$$

where each $f_{i}$ vector has unit $z$-component, and $\sum_{i=1}^{n} \alpha_{i}$ corresponds to the normal component of the contact force. In a common definition for the GWS computation, the normal component of each individual contact is limited to one [31]. Each force vector $f_{i}$ results in an object wrench that makes up the Grasp matrix. The Grasp matrix can be seen as the column space of wrenches contributed by all contacts. One of the most important properties of a grasp is its capability of inserting desired contact forces in a given grasp configuration.

The GWS for the grasp configuration can demonstrate the set of wrenches that the grasp could tolerate using the capability of inserting contact forces. The GWS for the illustrated grasp configuration included a neighborhood of the origin which showed its force closure property. Thus, the grasp can tolerate any external forces by applying suitable contact forces. For instance, looking at maximum normalized forces in $x$ direction in Fig. 10a,b, for friction coefficient 0.2 and 0.5 while assuming all contact forces are equal to $10 \mathrm{~N}$, the grasp can tolerate up to $1.9 \mathrm{~N}$ and $4.4 \mathrm{~N}$ external forces, respectively. An experiment was conducted to validate the strength of the grasp suggested by GWS in these figures.

The friction coefficient was measured to be 0.5 and $f_{c_{1}}$ and $f_{c_{3}}$ were regulated to be $10 \mathrm{~N}$. An external disturbance force set in $x$ direction $\left(f_{x d}\right)$ was applied. Fig. 11/ a shows the amount of force that was read until the grasp failed. As seen the grasp tolerated the maximum of $4.6 \mathrm{~N}$. This amount is slightly more $(4 \%)$ than what was expected since in our calculation it was assumed that the second phalanxes inserted equal contact forces. In the second experiment, $f_{c_{1}}=f_{c_{3}}$ were regulated at $30 \mathrm{~N}$ and this time tried to pull the object in the $z$ direction instead of $x$. Based on the calculated GWS the grasp was supposed to tolerate $30 \mathrm{~N}$. However, a small difference was observed (5\%), and the grasp tolerated the maximum of $28.5 \mathrm{~N}$ due to uncertainties in distal phalanx force approximation. Fig. 11p shows the amount of force that was read until the grasp was broken.

The structure of the fingers were evaluated to be capable of regulating up to a maximum of $78 \mathrm{~N}$ contact forces without any structural failure. This strength and the small finger size allow conducting grasps strong enough for many robotic applications including agriculture.

\section{E. Contact Point Estimation}

The proposed design allows for predicting contact points with an object and potentially predicting its shape. To validate this capability, a series of experiments with random shape objects were performed. As previously described, using the Jacobian matrix information, one can obtain joint torque vector using (5) and (7) as follows,

$$
K_{2} \Delta \theta_{2}=c_{1}^{2} f_{c_{1}}, K_{5} \Delta \theta_{5}=c_{3}^{2} f_{c_{3}}
$$




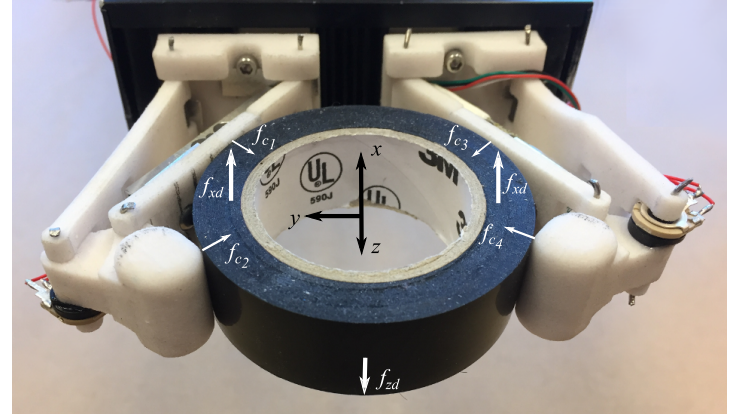

Fig. 9. Four-contact grasp configuration of a circular object.

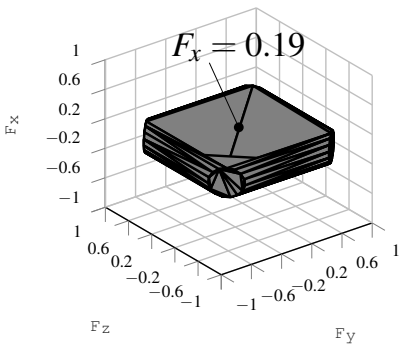

(a)

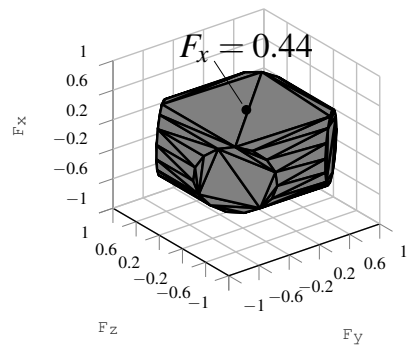

(b)
Fig. 10. Grasp Wrench Space (GWS). The friction cone was approximated with a 15 -sided pyramid. The approximation pyramid with more sides gives more accurate triangulation which results in better visual representation and higher accuracy of GWS. (a) GWS of the given four-contact grasp configuration in the presence of friction with 0.2 friction coefficient. The maximum normalized force in $x$ direction is pinned. (b) GWS of the given four-contact grasp configuration in the presence of friction with 0.5 friction coefficients. The maximum normalized force in $x$ direction is pinned.

where $K_{2} \Delta \theta_{2}$ and $K_{5} \Delta \theta_{5}$ are equal to the actuation torque of the two fingers, $f_{c_{1}}$ and $f_{c_{3}}$ are the first contact forces on the fingers, respectively. By regulating the second joint variables $\left(\theta_{2}\right.$ and $\left.\theta_{5}\right)$ and measuring the forces $\left(f_{c_{1}}\right.$ and $\left.f_{c_{3}}\right)$ using load cells, (9) can be verified.

A comparison between the measured values of contact points and those estimated using (9) is shown in Fig. 12 based on the graduated proximal phalanx in Fig. 4 In this experiment, the contact points along the proximal phalanx were estimated, using the corresponding values of the contact

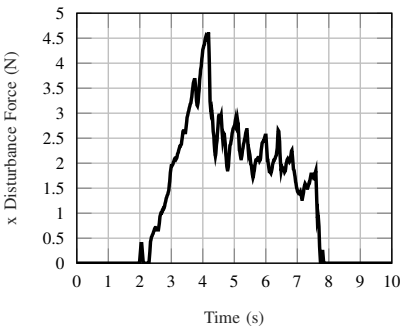

(a)

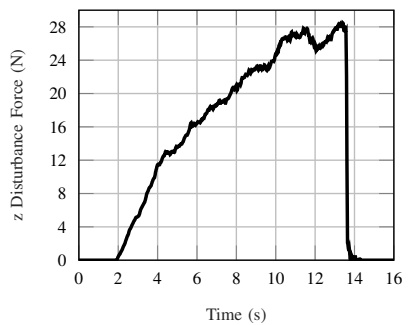

(b)
Fig. 11. Grasp external wrench tolerance. (a) Disturbance wrench tolerance in $x$ direction while $f_{c_{1}}=f_{c_{3}}=10 \mathrm{~N}$. (b) Disturbance wrench tolerance in $z$ direction while $f_{c_{1}}=f_{c_{3}}=30 \mathrm{~N}$.

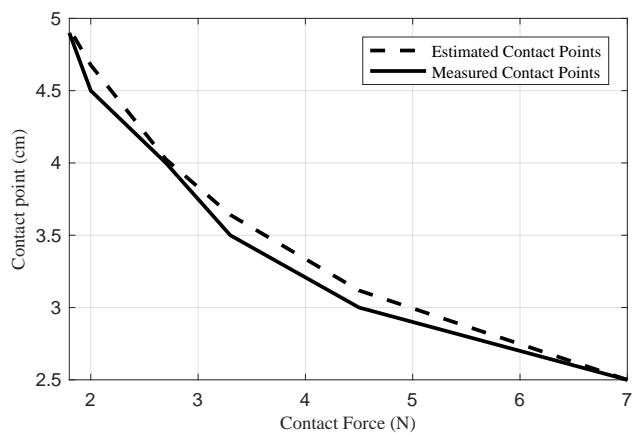

Fig. 12. Contact points estimation using measured contact forces on the proximal phalanx of the finger.

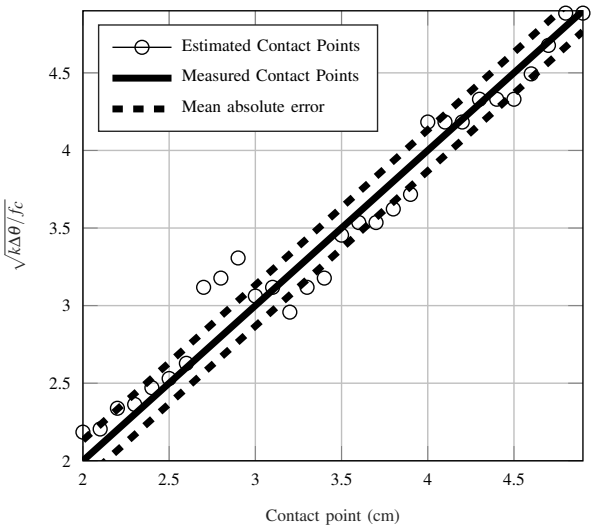

Fig. 13. Actual contact points and estimated values.

force. The second joint was regulated at $1.2 \mathrm{rad}$ while proximal phalanx was in contact with the object at different contact points. The results shows the validity of the contact point estimation.

To validate the accuracy of contact point estimation, 30 more experiments with different objects were conducted. The proposed method was able to compute a fairly precise estimation of the contact location with a mean absolute error of $1.6 \%$ $(1.3 \mathrm{~mm})$ and a standard deviation of $10 \%(8.2 \mathrm{~mm})$ measured during 30 experiments. Figure 13 illustrates the results for these experiments performed with different contact locations along the proximal phalanx. Dots within the dashed lines in this figure show an error smaller than the mean absolute error. The inconsistencies shown in Fig. 13 can be associated with practical limitations. First, the joint friction is not considered in this paper. Thus, with the simple model used here, a deviation from the theoretical model was inevitable. Second, the stiffness coefficients of the springs can create significant errors since the instantaneous torque reaction is proportional to them.

To complete the experimental validation, the influence of the preshaping [27] was studied by changing the location of the initial contact on the proximal phalanx for different angles (six different $c_{1}$, and four different $\theta_{2}$ ). Estimation errors were computed for 24 configurations (see Fig. 14). As seen, the estimation error is on average $8.7 \%$ and is not impacted by the preshaping.

The distal phalanx in the proposed design is $64 \%$ shorter 


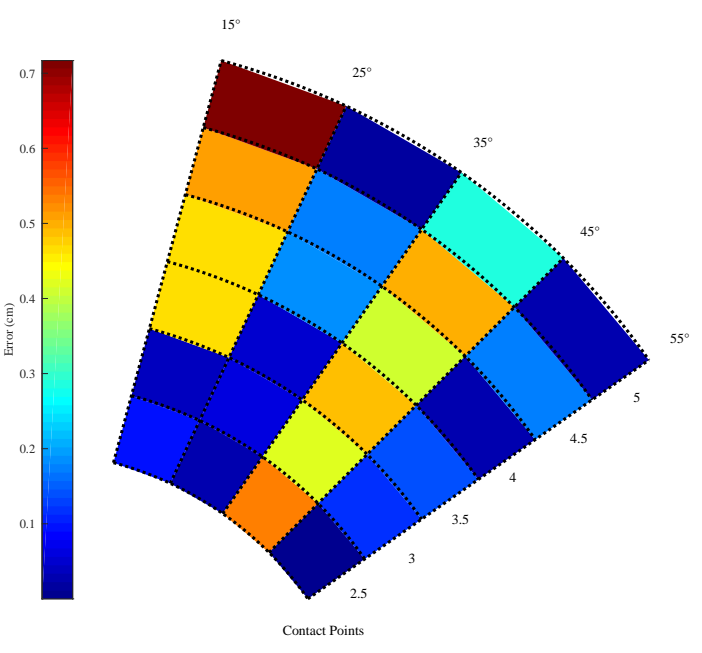

Fig. 14. Estimation error as a function of the initial contact position.

than the first one. As such, a contact point on the second phalanx has minimal torsional effect. The minimal leverage of contact forces along the second phalanx allows for assuming their contact points to be at the edge of this phalanx. This assumption eliminates the need for an additional load cell (or a tactile sensor) in the distal phalanx without compromising much accuracy. Further experiments using random convex and concave shapes validated this simplification.

To validate the simplification for the second phalanx contact point estimation, it is needed to quantify the amount of uncertainty it may cause in object position estimation and grasp analysis. The object frame is usually fixed to the centroid of the object to develop Grasp matrix or to be used for object placement. An experiment was designed in which finding the centroid of the object was desired. Different objects with general shapes were 3D printed and grasped. The contact forces on the proximal phalanx of each finger estimated using (9) and the second phalanx contact points were considered to be on the edge of the phalanx.

Kinematic dependency in all joints of the underactuated finger, as well as contact points knowledge, were used to approximate the grasped object with a polygon/polyhedron. Assuming that $\left\{c_{i}=\left(x_{i}, y_{i}\right)\right\}_{i=0}^{n_{c}} \subset R^{2}$ is a closed polygon in the plane, and the vertices are the contact points ordered counter clockwise. The centroid of the polygon is given by,

$$
p=\frac{1}{6 A}\left[\begin{array}{l}
\sum_{i=0}^{N-1}\left(x_{i}+x_{i+1}\right)\left(x_{i} y_{i+1}-x_{i+1} y_{i}\right) \\
\sum_{i=0}^{N-1}\left(y_{i}+y_{i+1}\right)\left(x_{i} y_{i+1}-x_{i+1} y_{i}\right)
\end{array}\right]
$$

where $A=\frac{1}{2} \sum_{i=0}^{N-1}\left(x_{i} y_{i+1}-x_{i+1} y_{i}\right)$, is the area enclosed by the polygon. Knowing all contact points on both fingers, the object shape was approximated with a 4-gon, and its centroid was obtained using (10). The shape matching derivation [32], however, was out of scope of this paper. Fig. 15 shows the validation of contact points and object center estimation for various convex and concave objects. As seen, the simplification made on the contact point estimation of the distal phalanx had insignificant effect (less than $3 \mathrm{~mm}$ ) on centroid estimation.

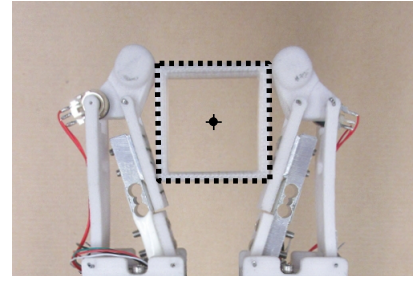

(a)

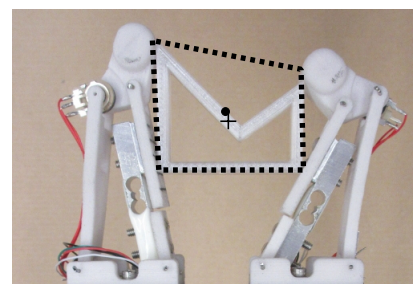

(c)

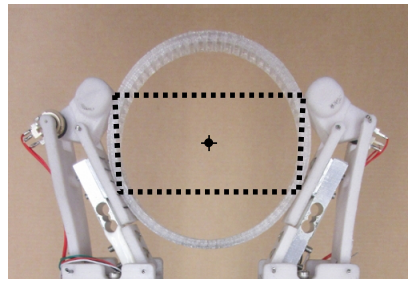

(b)

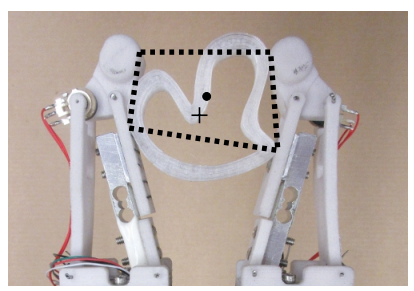

(d)
Fig. 15. Object centroid estimation. '•' delineates the estimated centroid, and '+' delineates the actual mass center. (a) square centroid and mass center, (b) circle centroid and mass center, (c) a concave polygon centroid and mass center, and (d) a concave object centroid and its mass center.

\section{DISCUSSION}

In this paper, a new design for a link-driven underactuated mechanism was proposed. In the proposed design a load cell and a potentiometer were able to provide enough data for contact force and position estimation. A prototype was built based on the proposed approach. The prototype was considered to have a relatively small size to be practical in a wide range of applications such as agriculture. Hence, only the proximal phalanx was considered to have embedded loadcell. This fact limited the capability of the finger for contact point estimation. As discussed before, a simplification for contact point estimation on the distal phalanx was proposed and validated on different objects. One should note that this is not a limitation of the proposed design method. Technically every phalanx of a link-driven underactuated mechanism can have a load cell in their structure for contact point estimation.

\section{CONCLUSION AND FUture WORK}

In this paper, Jacobian matrix, containing contact model and transmission matrix for an underactuated system with sensorized fingers were obtained. The proposed fingers were equipped with force and position sensors. The data from proprioceptive sensors were used to perform a wide range of tasks from power and precision grasping of both fragile and hard objects to estimating the shape and centroid of various concave and convex objects. The proposed design enjoys both compact and simple construction and provides a suitable alternative to those using tactile sensors. Experimental data using prototyped fingers were obtained from 67 different experimental scenarios to validate these claims. The future work will focus on fusing the data from the position and force sensor to have a better force regulation in the presence of nonlinear/anisotropic joint frictions and measurement noise that are inherent in all force sensors. 


\section{ACKNOWLEDGMENT}

The authors would like to thank S. Salarian for her valuable suggestions and L. Birglen for his helpful technical remarks. Financial support for this research was provided by the Natural Sciences and Engineering Research Council of Canada under a Collaborative Research and Develeopment grant (Grant No. 488611-15, M.R. Kermani Principal Investigator).

\section{REFERENCES}

[1] P. O. Hugo, "Industrial grippers: State-of-the-art and main design characteristics," in Grasping in Robotics. Springer, 2013, pp. 107-131.

[2] M. Abdeetedal and M. R. Kermani, "Grasp synthesis for purposeful fracturing of object," Robotics and Autonomous Systems, vol. 105, pp. $47-58,2018$.

[3] — - "Grasp evaluation method for applying static loads leading to beam failure," in Intelligent Robots and Systems (IROS), 2017 IEEE/RSJ International Conference on. IEEE, 2017, pp. 5999-6004.

[4] _ - "Optimal grasp synthesis to apply normal and shear stresses of failure in beams," in Advanced Intelligent Mechatronics (AIM), 2016 IEEE International Conference on. IEEE, 2016, pp. 395-400.

[5] S. Hirose and Y. Umetani, "The development of soft gripper for the versatile robot hand," Mechanism and machine theory, vol. 13, no. 3, pp. 351-359, 1978.

[6] A. M. Dollar and R. D. Howe, "The highly adaptive sdm hand: Design and performance evaluation," The international journal of robotics research, vol. 29, no. 5, pp. 585-597, 2010.

[7] F. Lotti, P. Tiezzi, G. Vassura, L. Biagiotti, G. Palli, and C. Melchiorri, "Development of ub hand 3: Early results," in Robotics and Automation, 2005. ICRA 2005. Proceedings of the 2005 IEEE International Conference on. IEEE, 2005, pp. 4488-4493.

[8] M. C. Carrozza, C. Suppo, F. Sebastiani, B. Massa, F. Vecchi, R. Lazzarini, M. R. Cutkosky, and P. Dario, "The spring hand: development of a self-adaptive prosthesis for restoring natural grasping," Autonomous Robots, vol. 16, no. 2, pp. 125-141, 2004.

[9] L. Birglen, T. Laliberté, and C. M. Gosselin, Underactuated robotic hands. Springer, 2007, vol. 40.

[10] M. Ciocarlie, C. Goldfeder, and P. Allen, "Dexterous grasping via eigengrasps: A low-dimensional approach to a high-complexity problem," in Robotics: Science and Systems Manipulation Workshop-Sensing and Adapting to the Real World. Citeseer, 2007.

[11] M. G. Catalano, G. Grioli, A. Serio, E. Farnioli, C. Piazza, and A. Bicchi, "Adaptive synergies for a humanoid robot hand," in 2012 12th IEEERAS International Conference on Humanoid Robots (Humanoids 2012). IEEE, 2012, pp. 7-14.

[12] M. Gabiccini, E. Farnioli, and A. Bicchi, "Grasp analysis tools for synergistic underactuated robotic hands," The International Journal of Robotics Research, p. 0278364913504473, 2013.

[13] T. Laliberté, L. Birglen, and C. Gosselin, "Underactuation in robotic grasping hands," Machine Intelligence \& Robotic Control, vol. 4, no. 3, pp. 1-11, 2002.

[14] M. Abdeetedal and M. R. Kermani, "Development and grasp analysis of a sensorized underactuated finger," in Intelligent Robots and Systems (IROS), 2017 IEEE/RSJ International Conference on. IEEE, 2017, pp. 6331-6336.

[15] J. Bohg, A. Morales, T. Asfour, and D. Kragic, "Data-driven grasp synthesis-a survey," IEEE Transactions on Robotics, vol. 30, no. 2, pp. 289-309, 2014.

[16] A. M. Dollar, L. P. Jentoft, J. H. Gao, and R. D. Howe, "Contact sensing and grasping performance of compliant hands," Autonomous Robots, vol. 28 , no. 1 , pp. 65-75, 2010.

[17] H. Yousef, M. Boukallel, and K. Althoefer, "Tactile sensing for dexterous in-hand manipulation in robotics-a review," Sensors and Actuators A: physical, vol. 167, no. 2, pp. 171-187, 2011.

[18] Y. Bekiroglu, J. Laaksonen, J. A. Jorgensen, V. Kyrki, and D. Kragic, "Assessing grasp stability based on learning and haptic data," IEEE Transactions on Robotics, vol. 27, no. 3, pp. 616-629, 2011.

[19] Z. Pezzementi, E. Plaku, C. Reyda, and G. D. Hager, "Tactileobject recognition from appearance information," IEEE Transactions on Robotics, vol. 27, no. 3, pp. 473-487, 2011.

[20] S. Shirafuji and K. Hosoda, "Detection and prevention of slip using sensors with different properties embedded in elastic artificial skin on the basis of previous experience," Robotics and Autonomous Systems, vol. 62 , no. 1 , pp. 46-52, 2014.
[21] F. L. Hammond, R. K. Kramer, Q. Wan, R. D. Howe, and R. J. Wood, "Soft tactile sensor arrays for micromanipulation," in 2012 IEEE/RSJ International Conference on Intelligent Robots and Systems. IEEE, 2012, pp. 25-32.

[22] Z. Kappassov, J.-A. Corrales, and V. Perdereau, "Tactile sensing in dexterous robot hands-review," Robotics and Autonomous Systems, vol. 74, pp. 195-220, 2015.

[23] M. C. Carrozza, F. Vecchi, F. Sebastiani, G. Cappiello, S. Roccella, M. Zecca, R. Lazzarini, and P. Dario, "Experimental analysis of an innovative prosthetic hand with proprioceptive sensors," in Robotics and Automation, 2003. Proceedings. ICRA'03. IEEE International Conference on, vol. 2. IEEE, 2003, pp. 2230-2235.

[24] G. S. Koonjul, G. J. Zeglin, and N. S. Pollard, "Measuring contact points from displacements with a compliant, articulated robot hand," in Robotics and Automation (ICRA), 2011 IEEE International Conference on. IEEE, 2011, pp. 489-495.

[25] S. B. Backus and A. M. Dollar, "Robust, inexpensive resonant frequency based contact detection for robotic manipulators," in Robotics and Automation (ICRA), 2012 IEEE International Conference on. IEEE, 2012, pp. 1514-1519.

[26] B. Belzile and L. Birglen, "Stiffness analysis of double tendon underactuated fingers," in Robotics and Automation (ICRA), 2014 IEEE International Conference on. IEEE, 2014, pp. 6679-6684.

[27] — "Stiffness analysis of underactuated fingers and its application to proprioceptive tactile sensing," IEEE/ASME Transactions on Mechatronics, vol. 21, no. 6, pp. 2672-2681, 2016.

[28] J. K. Davidson and K. H. Hunt, Robots and screw theory: applications of kinematics and statics to robotics. Oxford University Press on Demand, 2004.

[29] L. Birglen, "Enhancing versatility and safety of industrial grippers with adaptive robotic fingers," in Intelligent Robots and Systems (IROS), 2015 IEEE/RSJ International Conference on. IEEE, 2015, pp. 2911-2916.

[30] C. B. Barber, D. P. Dobkin, and H. Huhdanpaa, "The quickhull algorithm for convex hulls," ACM Transactions on Mathematical Software (TOMS), vol. 22, no. 4, pp. 469-483, 1996.

[31] C. Ferrari and J. Canny, "Planning optimal grasps," in Robotics and Automation, 1992. Proceedings., 1992 IEEE International Conference on. IEEE, 1992, pp. 2290-2295.

[32] Y. Li, J. L. Fu, and N. S. Pollard, "Data-driven grasp synthesis using shape matching and task-based pruning," IEEE Transactions on Visualization and Computer Graphics, vol. 13, no. 4, pp. 732-747, 2007.

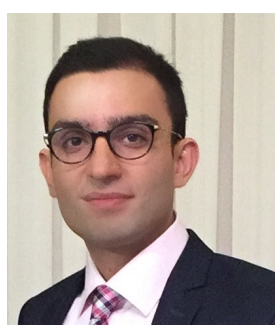

Mahyar Abdeetedal received the B.Sc. degree from the Shiraz University, Shiraz, Iran, in 2009, the M.Sc. degree from the Amirkabir University of Technology, Tehran, Iran, in 2012, Ph.D. degree from Western University, London, ON, Canada, in 2017, all in electrical engineering. His research interest includes agricultural robotics, robotic grasping, mechatronics.

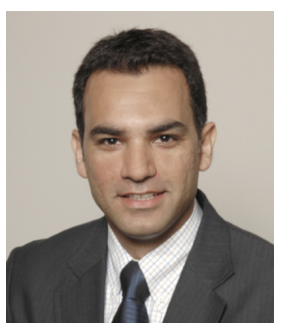

Mehrdad Radji Kermani received the B.Sc. degree from the Isfahan University of Technology, Isfahan, Iran, in 1994, the M.Sc. degree from the Iran University of Science and Technology, Tehran, Iran, in 1997, and the Ph.D. degree from the University of Western Ontario, London, ON, Canada, in 2005, all in electrical and computer engineering. From 1997 to 2001, he was a Senior Automation Consultant in the field of steel industries. He is currently an Associate Professor at the Department of Electrical and Computer Engineering, University of Western Ontario. His research interest includes human-safe robotic systems, smart materials, mechatronics, and actuation mechanisms. 